\title{
Activated parathyroid hormone/parathyroid hormone-related protein receptor in osteoblastic cells differentially affects cortical and trabecular bone
}

\author{
L.M. Calvi, ${ }^{1}$ N.A. Sims, ${ }^{2}$ J.L. Hunzelman, ${ }^{1}$ M.C. Knight,${ }^{1}$ A. Giovannetti, ${ }^{1}$ J.M. Saxton, ${ }^{1}$ \\ H.M. Kronenberg, ${ }^{1}$ R. Baron, ${ }^{2}$ and E. Schipani ${ }^{1}$ \\ ${ }^{1}$ Endocrine Unit, Massachusetts General Hospital and Harvard Medical School, Boston, Massachusetts, USA \\ ${ }^{2}$ Yale University, School of Medicine, New Haven, Connecticut, USA
}

Address correspondence to: E. Schipani, Endocrine Unit, Massachusetts General Hospital and Harvard Medical School, 50 Blossom Street, Boston, Massachusetts 02114, USA.

Phone: (617) 726-3966; Fax: (617) 726-7543; E-mail: schipani@helix.mgh.harvard.edu.

Received for publication September 11, 2000, and accepted in revised form December 18, 2000.

Parathyroid hormone (PTH), an important regulator of calcium homeostasis, targets most of its complex actions in bone to cells of the osteoblast lineage. Furthermore, PTH is known to stimulate osteoclastogenesis indirectly through activation of osteoblastic cells. To assess the role of the PTH/PTHrelated protein receptor (PPR) in mediating the diverse actions of PTH on bone in vivo, we generated mice that express, in cells of the osteoblastic lineage, one of the constitutively active receptors described in Jansen's metaphyseal chondrodysplasia. In these transgenic mice, osteoblastic function was increased in the trabecular and endosteal compartments, whereas it was decreased in the periosteum. In trabecular bone of the transgenic mice, there was an increase in osteoblast precursors, as well as in mature osteoblasts. Osteoblastic expression of the constitutively active PPR induced a dramatic increase in osteoclast number in both trabecular and compact bone in transgenic animals. The net effect of these actions was a substantial increase in trabecular bone volume and a decrease in cortical bone thickness of the long bones. These findings, for the first time to our knowledge, identify the PPR as a crucial mediator of both bone-forming and bone-resorbing actions of PTH, and they underline the complexity and heterogeneity of the osteoblast population and/or their regulatory microenvironment.

J. Clin. Invest. 107:277-286 (2001).

\section{Introduction}

In bone remodeling the activities of osteoblasts, the bone-forming cells, and osteoclasts, cells of hematopoietic origin capable of resorbing bone, must be balanced carefully in order to maintain skeletal integrity (1). The importance of understanding the factors controlling these activities is highlighted by metabolic bone disorders such as osteoporosis, in which the imbalance of bone formation and resorption leads to bone loss. Parathyroid hormone (PTH), a major regulator of calcium homeostasis, plays an important role in both bone formation and resorption. While PTH excess in hyperparathyroidism (2) and in continuous administration of PTH (3) is characterized by large numbers of osteoclasts, rapid bone turnover, and low cortical bone mass, it has long been known that intermittent dosing of PTH can lead to increased trabecular bone mass $(4,5)$. This anabolic effect is due to increased bone formation $(6,7)$. Interestingly, histomorphometric studies in patients with mild hyperparathyroidism also show an increase in cancellous bone (2). Although osteoblasts likely mediate both the anabolic and catabolic actions of the peptide, the molecular mechanisms underlying this dual effect are incompletely understood.
The PTH/PTH-related protein (PTH/PTHrP) receptor (PPR), a $G$ protein-coupled receptor, is believed to mediate many of the actions of both PTH and PTHrP in bone, as shown by mutations in mice and humans. Mice in which the PPR has been ablated by homologous recombination have decreased trabecular bone and increased thickness of cortical bone during fetal development (8). These skeletal abnormalities are similar to those observed in patients with Blomstrand lethal chondrodysplasia, a rare autosomal recessive disorder caused by inactivating PPR mutations $(9,10)$. Consistent with this crucial role of the PPR in cells of the osteoblast lineage, expression of the mRNA encoding this receptor is detectable in relatively mature osteoblasts and in adjacent stromal cells likely to be osteoblast precursors (11).

Jansen's metaphyseal chondrodysplasia (JMC) is a rare form of short-limbed dwarfism caused by activating mutations of the PPR leading to ligand-independent cAMP accumulation (12). Histomorphometric analysis of bone from a patient with this disorder shows exaggerated loss of cortical bone and preservation, or even augmentation of trabecular bone, as is seen in mild primary hyperparathyroidism (13). 
In the present study we generated mice that express in cells of the osteoblastic lineage, under the control of the $2.3-\mathrm{kb}$ fragment of the mouse $\alpha 1$ (I) collagen promoter, one of the human mutant PPRs described in JMC. The goal of our study was to use this PPR mutant as a tool in vivo to determine which actions of PTH can be mimicked solely by activating the PPR in osteoblasts. This study demonstrates that the PPR, when expressed only in cells of the osteoblastic lineage, can mediate both the anabolic and catabolic actions of PTH in bone.

\section{Methods}

Generation and identification of transgenic mice. The 2.3-kb fragment of the mouse $\alpha 1(\mathrm{I})$ collagen promoter (14) was excised from the plasmid pJ251, kindly provided by Benoit de Crombrugge (University of Texas, M.D. Anderson Cancer Center, Houston, Texas, USA), by double digestion with $\mathrm{XboI} / \mathrm{XbaI}$. The ends of this fragment were made blunt with Klenow polymerase and ligated to a blunt-ended BamHI site in the pcDNAI plasmid containing the cDNA that encodes the entire coding region of the human mutant PPR (HKrkH223R) (12), placing the promoter fragment upstream of HKrk-H223R. Restriction endonuclease digestions and nucleotide sequence analysis confirmed the correct orientation of the construct (Figure 1a). Nucleotide sequence analysis also confirmed the presence of an inframe stop codon and of the native Kozak consensus sequence upstream of the translation initiation codon. The construct insert, containing the $2.3-\mathrm{kb}$ fragment of the mouse $\alpha 1(\mathrm{I})$ collagen promoter, $1,880 \mathrm{bp}$ encoding HKrk-H223R and $750 \mathrm{bp}$ from the pcDNAI vector (which provides a splice sequence and the consensus polyadenylation signal absent in the cDNA encoding HKrk-H223R), was released from the vector by digestion with HindIII and ScaI (Figure 1a) and purified according to standard techniques. Microinjections into the pronucleus of fertilized oocytes were performed at the Massachusetts General Hospital Transgenic Facility (Boston, Massachusetts, USA). Founder mice of the FVB/N strain were mated with wild-type mice to establish individual transgenic lines. Genotyping and determination of the number of insertion sites of the transgene were performed as described (15). All studies performed were approved by the institutional animal care committee. Assessment of transgene expression. To assess the expression of the mutant PPR transgene in bone, total RNA was extracted from newborn calvaria using TRIzol reagent (Life Technologies, Gaithersburg, Maryland, USA). Total RNA was reversed transcribed into singlestranded DNA using the pcDNAI-specific reverse primer A (5'-TTCCACCACTGCCTCCCATTCATC-3') and the Omniscript Reverse Transcriptase kit (QIAGEN Inc., Valencia, California, USA). PCR was performed using the same reverse primer $\mathrm{A}$ and the forward primer S1 (5'-CACCTGCCCTGCTACAGGAAGAG-3') (Figure 1a) with the following conditions: $94^{\circ} \mathrm{C}$ for 1 minute, $58^{\circ} \mathrm{C}$ for 45 seconds, $72^{\circ} \mathrm{C}$ for 1 minute, and an additional 10 minutes at $72^{\circ} \mathrm{C}$ at the end of the 35 cycles. In addition, the PCR products were determined by Southern blot analysis using the ${ }^{32} \mathrm{P}$-labeled oligonucleotide S (5'-TGACATAGTGGATGGACAGATGG$\left.3^{\prime}\right)$. To confirm transgene expression by in situ hybridization, a 596-bp probe (DT7) was generated by PCR amplification of the pcDNAI vector sequence in the transgene construct using the reverse primer A1 (5'-TAATACGACTCACTATAGGGCGATAAACAAGTTAACAACAACAAT- $\left.3^{\prime}\right)$ and the forward primer S2 $\left(5^{\prime}\right.$ CTTTGTGAAGGAACCTTACT- $3^{\prime}$ ) (Figure 1a). The A1 reverse primer sequence also includes the T7 RNA polymerase-binding site. The PCR conditions were as follows: $94^{\circ} \mathrm{C}$ for 1 minute, $58^{\circ} \mathrm{C}$ for 45 seconds, $72^{\circ} \mathrm{C}$ for 1 minute, and an additional 10 minutes at $72^{\circ} \mathrm{C}$ at the end of the 45 cycles.

Serology. Serum was collected at 1 month of age for ionized calcium and PTH-level determination. Ionized calcium was measured by the Ciba-Corning 634 $\mathrm{Ca}^{++} / \mathrm{pH}$ analyzer (Ciba-Corning Diagnostics Corp., Medfield, Massachusetts, USA). Intact immunoreactive PTH was measured in duplicate using ELISA (Immutopics Inc., San Clemente, California, USA), which uses two affinity-purified polyclonal Ab's raised to peptides common to rat and mouse PTH. The first Ab recognizes epitopes within the midregion/carboxy-terminal portion of the peptide (region 39-84), while the second $\mathrm{Ab}$, conjugated to horseradish peroxidase for detection, recognizes epitopes in the amino-terminal region (region 1-34). Statistical analysis was performed using the Student's $t$ test, and $P$ values less than 0.05 were accepted as significant.

Sample preparation and histologic analysis. For histologic analysis, transgenic mice and sex-matched wild-type littermates were sacrificed by cervical dislocation at birth, $1,2,4$, and 12 weeks of age. Tissues from transgenic and wild-type mice were fixed and stored as described (15). In selected cases, hindlimbs and/or skulls were decalcified (15), and paraffin blocks were prepared by standard histological procedures. For selected samples, tartrate-resistant acid phosphatase (TRAP) staining was performed using a Sigma Chemical Co. acid phosphatase detection kit (St. Louis, Missouri, USA).

Histomorphometry. Tibiae and calvaria were collected for histomorphometric analysis at 2, 6, and 12 weeks of age. For dynamic histomorphometry, 12-week-old animals were injected intraperitoneally with fluorochromes: calcein $(2 \mathrm{mg} / \mathrm{ml}$, Sigma Chemical Co.) and demeclocycline $(2 \mathrm{mg} / \mathrm{ml}$, Sigma Chemical Co.) were injected 3 and 10 days before sacrifice, respectively. Bones were fixed and embedded in methyl methacrylate resin as described previously (16). Five-micrometer sections were stained with toluidine blue or coverslipped unstained, and histomorphometric analysis was performed with the Osteomeasure system (Osteometrics Inc., Atlanta, Georgia, USA) using standard procedures (17). Tibial sections were measured in the proximal metaphysis beginning $340 \mu \mathrm{m}$ below the chondro-osseous junction. Tibial cortical thickness and periosteal mineral appositional rates were meas- 
ured beginning $680 \mu \mathrm{m}$ from the chondro-osseous junction on the anterofibular side. Coronal sections of the parietal bone were measured immediately frontal to the interparietal bone, excluding $300 \mu \mathrm{m}$ on either side of the sagittal suture. Statistical analysis was performed using ANOVA, with $P$ values less than 0.05 accepted as significant; error bars represent \pm SEM.

In situ bybridization. In situ hybridizations were performed as described (15) using complementary ${ }^{35} \mathrm{~S}$ labeled riboprobes (complementary RNAs and cRNAs) transcribed from the plasmids encoding rat alkaline phosphatase (nucleotides 1-241; GenBank accession no. J03572), collagenase 3 (18), human pro-alpha 1(I) chain of type I procollagen (nucleotides 2361-3347; GenBank accession no. K01228), mouse 92-kDa type IV collagenase or MMP-9 (nucleotides 1945-2266), mouse osteopontin (nucleotides 157-1144; GenBank accession no. X14882), rat PPR (nucleotides 1-2065; GenBank accession no. M77184), and rat TRAP (nucleotides 1-832; GenBank accession no. M76110). In addition, the complementary ${ }^{35}$ S-labeled riboprobe transcribed from the DT7 PCR product was used to detect expression of the transgene mRNA.

Analysis of bromodeoxyuridine incorporation. Mutant and transgenic mice at 1 and 2 weeks of age were injected intraperitoneally with $100 \mu \mathrm{g}$ bromodeoxyuridine (BrdU) $/ 12 \mu \mathrm{g}$ fluorodeoxyuridine (FdU) per gram of body weight 2 hours before sacrifice. After sacrifice, hindlimbs were dissected, fixed, decalcified, and embedded in paraffin, and longitudinal sections across the tibia and femur were obtained. To identify actively proliferating cells, nuclei that had incorporated BrdU were detected using a Zymed BrdU immunostaining kit (Zymed Laboratories Inc., South San Francisco, California, USA). For each section, high-power field images of the primary spongiosa were photographed. All BrdU-positive (black) and BrdU-negative (pink) nuclei in this zone were counted. One or two nonconsecutive sections were counted for each of two or three wild-type and two or three transgenic specimens at each time point. To assess proliferation rates in periosteal cells, all BrdU-positive and BrdU-negative nuclei were counted in two high-power field $(\times 400)$ images from equivalent portions of the lateral periosteum.

Determination of apoptotic osteoblasts in vivo. Apoptotic osteoblasts were detected in sections of decalcified tibiae of 2-week-old transgenic and wild-type littermates by the terminal deoxynucleotidyl transferase-mediated nick-end labeling (TUNEL) reaction using reagents from the ApopTag Plus Peroxidase In Situ Detection Kit (Intergen Co., Purchase, New York, USA). The sections were counterstained with methyl green. In each TUNELlabeling assay, sections of weaned rat mammary tissue were used as positive control. Negative controls were pre- $\mathbf{a}$

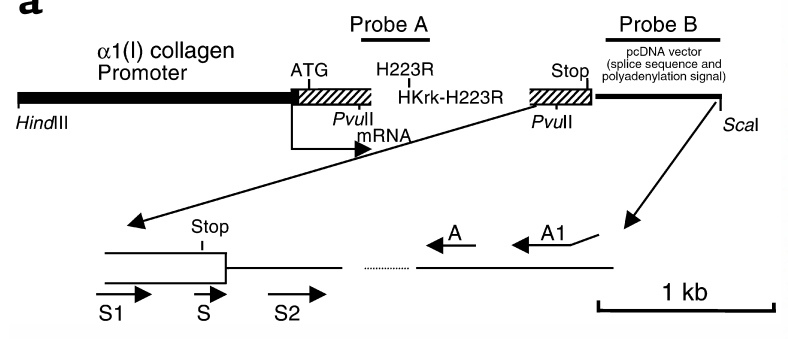

b

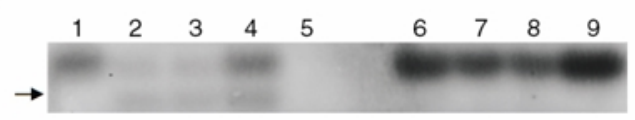

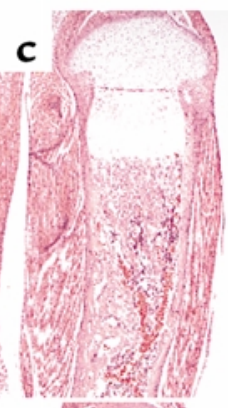

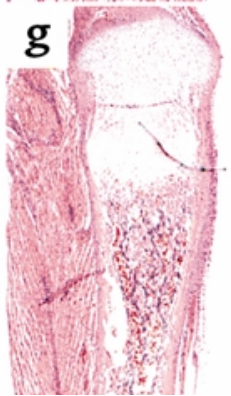

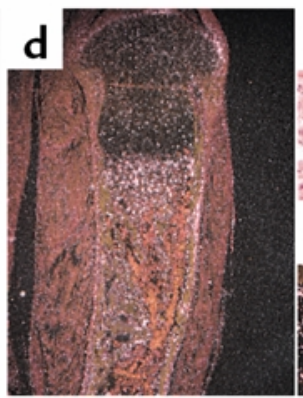
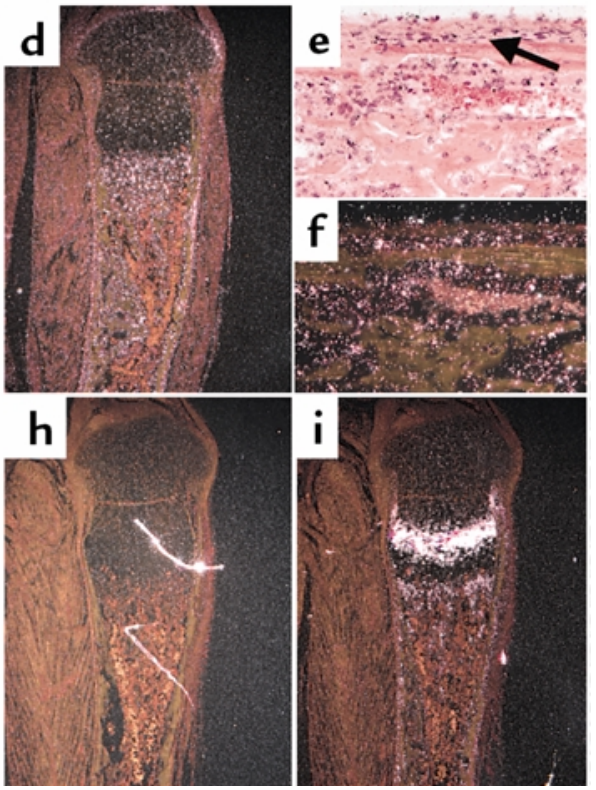

\section{Figure 1}

Transgene construct and its expression. (a) Shown is the scheme of the transgene construct with the location of probes A and B for Southern blot analysis of genomic DNA. Locations of the translation initiation codon (ATG), the receptor mutation (H223R), and the stop codon (Stop) in the cDNA encoding the human PPR mutant, HKrk-H223R, are seen. Restriction sites for the enzymes HindIII, Pvull, and Scal are also indicated. Oligonucleotide S was used as probe for Southern blot analysis of RT-PCR products, oligonucleotides S1 and A were used for RT-PCR of total RNA, and oligonucleotides S2 and A1 were used to generate the DT7 probe. (b) Southern blot analysis of the RT-PCR products with probe S: RT-PCR products from line not expressing the transgene (lanes 1 and 6 ), CL1 (lanes 2 , 3, 7, and 8), and CL2 (lanes 4 and 9), with negative control in lane 5 and -RT controls in lanes 6-9. (c-h) In situ hybridization with the ${ }^{35}$ S-labeled DT7 cRNA in serial section of decalcified proximal tibia of newborn CL2 mouse (c and $\mathbf{d}$ ), and a wild-type littermate ( $\mathbf{g}$ and h). In the high-magnification images from 2-week-old CL2 mutant (e and f), the arrow indicates the periosteal surface. (i) In situ hybridization with the ${ }^{35} \mathrm{~S}$-labeled PPR cRNA in a section of decalcified proximal tibia of a newborn wild-type mouse. All sections were counterstained with hematoxylin and eosin; bright-field (c, e, g) and dark-field (d, f, h, i) views are shown. 

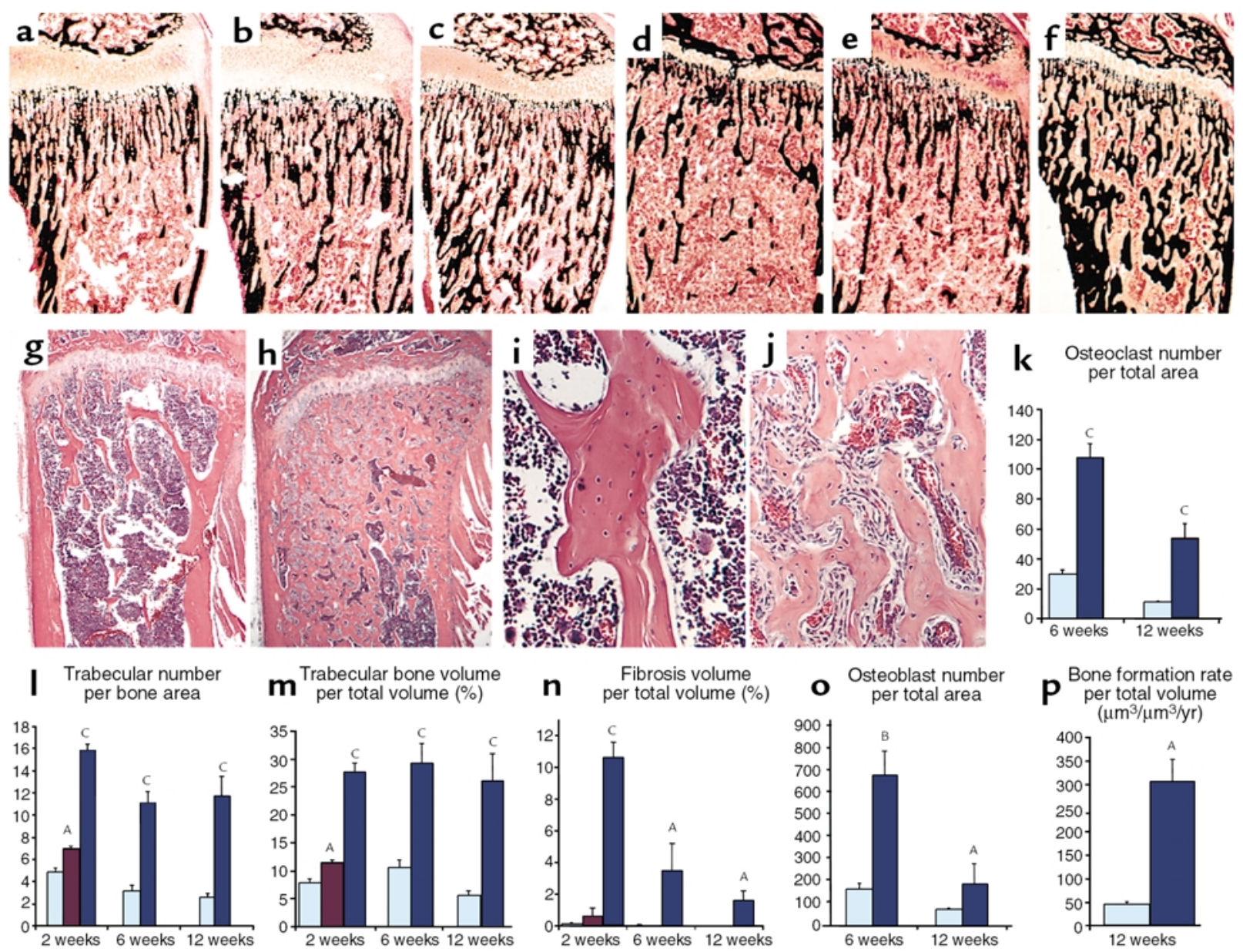

Figure 2

Histology and histomorphometry of trabecular bone. (a-f) Histologic sections of undecalcified proximal tibia of 2-week-old (a-c) and 6-week-old (d-f) wild-type ( $\mathbf{a}$ and $\mathbf{d}$ ), CL1 (b and $\mathbf{e}$ ), and CL2 (c and $\mathbf{f})$ mice stained by the method of Von Kossa. (g-j) Histologic sections, stained with hematoxylin and eosin, of decalcified proximal tibia from 12-week-old wild-type (g) and CL2 (h) littermates. In the high-power images from the proximal tibia of 12-week-old wild-type (i) and CL2 transgenic (j) littermates, the metaphyseal area is shown. (k-p) Histomorphometric analysis of wild-type (light blue), CL1 (magenta), and CL2 (dark blue) littermates. Ages of animals are indicated on the $x$-axis. ${ }^{A} P<0.05,{ }^{B} P<0.01,{ }^{C} P<0.005$. Error bars represent the SEM.

pared by omitting the transferase. As in the analysis of BrdU incorporation, two high-power field images of the primary spongiosa were photographed for each animal. All positive (brown) and negative (green) nuclei in this zone were counted. One section was counted for each of four wild-type and five transgenic specimens.

\section{Results}

Generation, identification, and gross phenotype of transgenic mice. Two transgenic lines (CL1 and CL2) expressing the constitutively active human PPR, HKrk-H223R (12), under the control of the $2.3-\mathrm{kb}$ fragment of the mouse $\alpha 1$ (I) collagen promoter (14) were established. Founder CL1 was shown by Southern blot analysis to carry only one copy of the transgene, while founder CL2 carried several copies (data not shown).

PCR analysis of reverse-transcribed RNA extracted from newborn calvaria revealed specific expression of the transgene. Southern blot analysis of PCR products showed a $280 \mathrm{bp}$-specific band, not present in the -reverse transcriptase (-RT) controls (Figure 1b); an additional 350-bp band was present in both $+\mathrm{RT}$ and -RT samples (Figure 1b), presumably as a result of amplification of genomic DNA contaminating the total RNA preparation. Expression of the transgene was detected in both lines. Relative levels of expression in the two lines were assessed by Northern blot analysis using probe DT7 and were noted to be many fold higher for the CL2 line (data not shown). For this line, transgene expression was also detected by in situ hybridization at birth and postnatally and localized to osteoblastic and stroma-like cells in the trabecular bone compartment, as well as the endosteal and periosteal surfaces of mutant bones of all ages tested (Figures 1, $\mathrm{c}-\mathrm{h}$, and data not shown). The pattern of expression of the transgene in mutant animals overlapped with that of the native PPR in wild-type animals in the osseous regions of the bone, but was slightly 
more diffuse, consistent with targeting by the $2.3-\mathrm{kb}$ fragment of the mouse $\alpha 1$ (I) collagen gene promoter (Figure 1i). In addition, low-level expression of the transgene was noted in the growth plate and at the site of attachment of tendons in sections from newborn transgenic mice (Figure 1d), although the characteristic pattern of high-level expression of the PPR in the early hypertrophic region of the growth plate was not seen (Figure 1i). No transgene expression was seen in multinucleated cells in the bones of CL2 transgenic animals (data not shown). Expression of the transgene could not be detected by in situ hybridization in the CL1 line (data not shown).

At birth, transgenic mice were indistinguishable from wild-type littermates. At 2 weeks of age, hemizygous transgenic mice from the CL1 line were very similar to normal mice, while homozygous mice had shortening of the limbs and the tail. Consistent with the higher level of transgene expression, 2-week-old hemizygous animals from the CL2 line were clearly smaller and had shorter limbs than sex-matched control littermates (data not shown). Therefore, homozygous animals from the CL1 line and hemizygous animals from the CL2 line were studied after birth. Both transgenic lines showed qualitatively similar skeletal changes at all stages of postnatal development studied in both males and females. In addition, consistent with high levels of expression of the transgene in odontoblasts, CL2 mice had abnormally large incisors, which erupted normally (data not shown).

Serology. There were no differences in ionized calcium between normal and transgenic mice of both lines (ionized $\mathrm{Ca}^{2++}$, millimoles per liter: wild-type, $n=12,1.30 \pm 0.02$; CL1, $n=4,1.33 \pm 0.03, P=0.624$; $\mathrm{CL} 2, n=6,1.29 \pm 0.03, P=0.929)$. Intact PTH measurements showed a slightly decreased PTH level in mutant compared with normal mice (PTH in picograms per milliliter: wild-type, $n=38,35.3 \pm 3.3$; CL2, $n=18,22.7 \pm 4.4, P<0.05$ ).

Trabecular bone. Histologic analysis of the skeleton in CL2 mice revealed an increase in trabeculation of the metaphyseal regions of the long bones. This trabeculation had a disorganized, reticular pattern, and it extended into the diaphysis with partial obliteration of the bone marrow cavity (Figure 2, g-h). The increase was present in sections from 1-week-old animals (data not shown), was very dramatic at 2 weeks (Figure 2 , a and c), and persisted in adulthood (Figure 2, $\mathrm{d}$ and $\mathrm{f}-\mathrm{h}$ ). In 1-week-old and 2-week-old mutants, the trabeculae close to the growth plate contained remnants of carti-
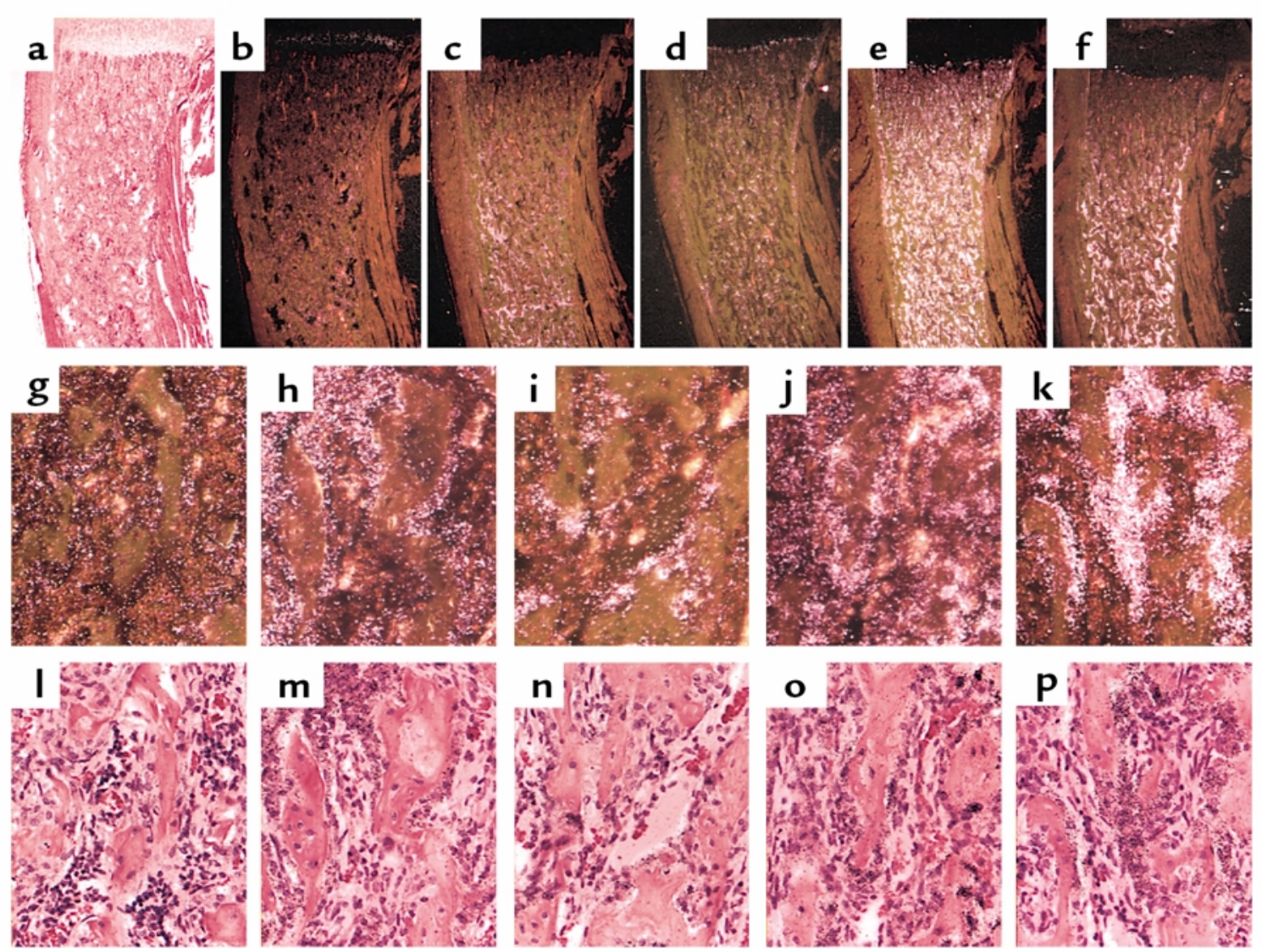

Figure 3

In situ hybridization analysis of trabecular bone. In situ hybridization with the ${ }^{35}$ S-labeled alkaline phosphatase $(\mathbf{a}, \mathbf{b}, \mathbf{g}, \mathbf{I})$, collagen I (c, h, $\mathbf{m})$, collagenase $3(\mathbf{d}, \mathbf{i}, \mathbf{n})$, osteopontin $(\mathbf{e}, \mathbf{j}, \mathbf{o})$, and osteocalcin $(\mathbf{f}, \mathbf{k}, \mathbf{p})$ cRNAs in serial sections of decalcified proximal tibia from 2-weekold CL2 mouse. Higher-magnification images of the trabecular area are shown $(\mathbf{g}-\mathbf{p})$. The sections were counterstained with hematoxylin and eosin; bright-field (a, I-p) and dark-field (b-k) views are shown. 


\section{Figure 4}

Osteoclast number and function. (a-d) In situ hybridization with the ${ }^{35}$ S-labeled TRAP cRNA in serial sections of decalcified proximal tibia of 2-week-old wild-type ( $\mathbf{a}$ and $\mathbf{b}$ ) and CL2 (c and $\mathbf{d})$ littermates. The sections were counterstained with hematoxylin and eosin; bright-field (a and c) and dark-field (b and $\mathbf{d}$ ) views are shown. (e and $\mathbf{f}$ ) High-power $(\times 200)$ light microscopy of enzymatic staining for TRAP activity in sections of decalcified proximal tibia from a 2-week-old CL2 mouse, focusing on the metaphyseal trabecular bone (e) and the diaphyseal cortical bone (f); the arrow indicates one of the TRAP-positive multinucleated cells. Sections were counterstained with methyl green.
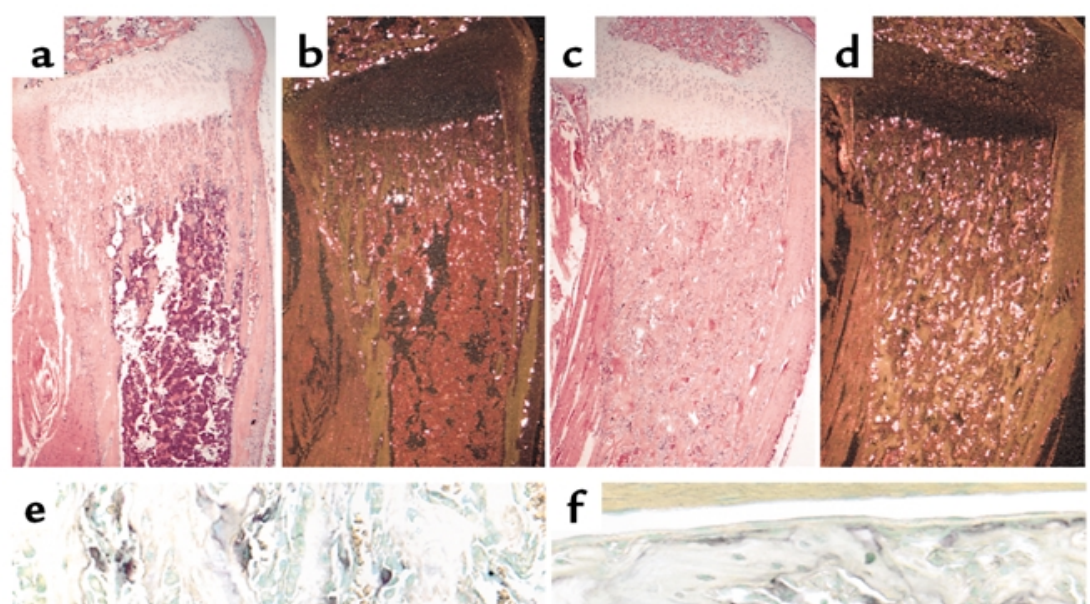

f

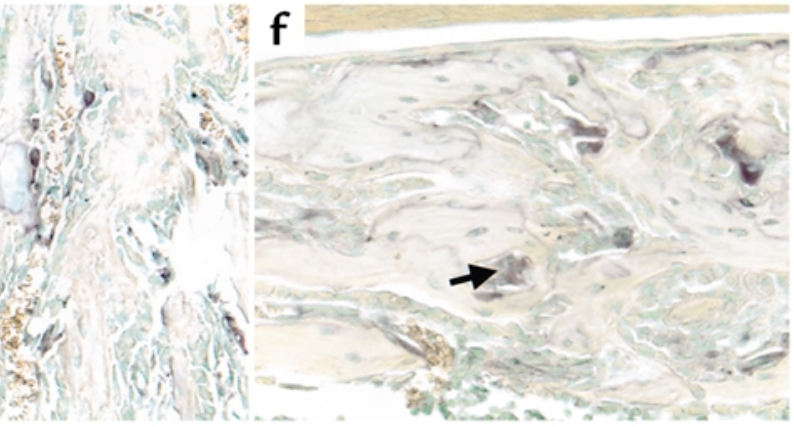

lage matrix as in a typical primary spongiosa, while no residual cartilage matrix was noted in the trabeculae that extended into the medullary cavity, suggesting that these trabeculae were the result of increased de novo bone formation and increased bone remodeling, rather than of defective osteoclast bone-resorbing activity (data not shown). The trabeculae were normally mineralized, as shown by Von Kossa staining (Figure 2, a-f). Histomorphometric analysis confirmed the increase in trabecular number and volume (Figure 2, 1 and $\mathrm{m}$ ). Trabecular thickness was unchanged (data not shown). In the transgenic mice, the spaces among the trabeculae, usually occupied by hematopoietic cells in wild-type animals, were populated mainly by stromalike cells with a fibrotic appearance (Figure $2, i$ and $j$ ), as confirmed by histomorphometric analysis (Figure 2n). A similar but milder phenotype was noted in CL1 mice (Figure 2, b, e, l, and $\mathrm{m}$ ).

To assess whether the increased trabecular bone volume seen in mutant mice could be secondary to augmented osteoblast activity, dynamic histomorphometry was performed. In 12 -week-old animals, there was a 6.5 -fold increase in trabecular bone formation rate compared with wild-type littermates $(P<0.05$, Figure $2 \mathrm{p})$. Trabecular mineral apposition rate, an index of osteoblast function, was also increased by $30 \%$ in transgenic mice compared with normal littermates (wild-type, micrometers per day: $0.968 \pm 0.08$; CL2: $1.253 \pm 0.04, P<0.05$ ). In addition, histomorphometric analysis showed a dramatic increase in osteoblast number (Figure 2o).

In situ hybridization analysis was performed to characterize the degree of differentiation of the stroma-like cells in the trabecular compartment of CL2 transgenic mice. Osteocalcin mRNA was expressed strongly by the cuboidal cells lining the trabeculae (Figure $3, \mathrm{f}, \mathrm{k}$, and p), suggesting they are fully mature osteoblasts (19). In addition, alkaline phosphatase (Figure 3, a, b, g, and l), collagen I (Figure 3, c, h, and $\mathrm{m}$ ), collagenase 3 (Figure $3, \mathrm{~d}, \mathrm{i}$, and $\mathrm{n}$ ), osteopontin (Figure $3, \mathrm{e}, \mathrm{j}$, and o) mRNAs were expressed heterogeneously by the stroma-like cells among the trabeculae, suggesting that these cells belong to the osteoblast lineage.

To assess whether changes in proliferation rate contributed to the expansion of the osteoblastic population, BrdU incorporation rates were measured in the trabecular compartment of 2-week-old wild-type and CL2 mutant animals. Proliferation rates were increased by $30 \%$ in transgenic animals (wild-type percentage $14.5 \pm 1.6$; CL2 $18.7 \pm 0.8, P<0.05)$. An even larger increase in proliferation rate was seen at 1 week of age (wild-type percentage $16.4 \pm 3.3$; CL2 $32.3 \pm 5.8$, $P<0.05)$. Conversely, in the same areas, the proportion of osteoblastic cells undergoing apoptosis, as detected by TUNEL labeling, was decreased in CL2 transgenic mice $(0.4 \% \pm 0.1)$ compared with wild-type littermates $(1.6 \% \pm 0.4, P<0.05)$, suggesting that changes in both proliferation and apoptosis rates contributed to the expansion of the osteoblast pool.

Histologic analysis of the long bones from mutant mice showed a dramatic increase in multinucleated cells, which was confirmed by histomorphometry (Figure $2 \mathrm{k}$ ). Many of these multinucleated cells expressed high levels of TRAP mRNA and protein, as shown by in situ hybridization analysis (Figure $4, a-d$ ) and specific enzymatic staining for TRAP activity (Figure 4e) and were therefore mature osteoclasts.

Cortical bone. Transgenic cortical bone in both CL1 and CL2 mice was histologically normal at birth (Fig- 
ure 1, $\mathrm{c}$ and $\mathrm{g}$, and data not shown). By 12 weeks of age, the predominant histological feature of the mutant CL2 cortical bone was its decreased thickness (Figure $5, \mathrm{c}-\mathrm{d}$ ), confirmed by histomorphometry (Figure 5e). No significant difference in cortical thickness could be observed in the CL1 mice, consistent with the milder skeletal phenotype of this line. Starting at 1 month of age in both CL1 and CL2 animals, the cortical bone was porous rather than compact (Figure 5, a-d), suggesting an increased bone-modeling/remodeling process at the endosteal surface. A large number of osteoclasts were present in the cortical bone of the mutant mice (Figure 4f).

Since periosteal mineral apposition rate is a major determinant of cortical bone formation and maintenance, this parameter was measured in 12-week-old wild-type and CL2 transgenic animals by dynamic histomorphometry. In striking contrast to the significant increase in trabecular mineral apposition rate seen in the transgenic mice, no periosteal mineral apposition rate could be measured in tibiae from the same transgenic animals (Figure 5f). To assess whether the presence of the transgene in periosteal cells also modified their proliferation rate, BrdU incorporation rate was measured in the periosteum. In contrast to the significant increase in proliferation rate seen in cells in the trabecular compartment, a 50\% decrease in BrdU incorporation was measured in the periosteal compartment of CL2 transgenic mice compared with wild-type littermates (wild-type $6.8 \pm 1.5 \%$; transgenic $3.5 \pm 0.5 \%$, $P<0.05)$. Characterization of the transgenic periosteal cells by in situ hybridization revealed a decreased level of expression of osteoblast markers such as alkaline phosphatase, collagen I and osteocalcin, in comparison with wild-type periosteal cells (Figures 5, e-p). The same markers appeared to be well expressed by the endosteal cells in both wild-type and mutant bones (Figure 5, e-p). Taken together, these data suggest that transgene expression has an inhibitory effect in the periosteum, but not in the endosteum.
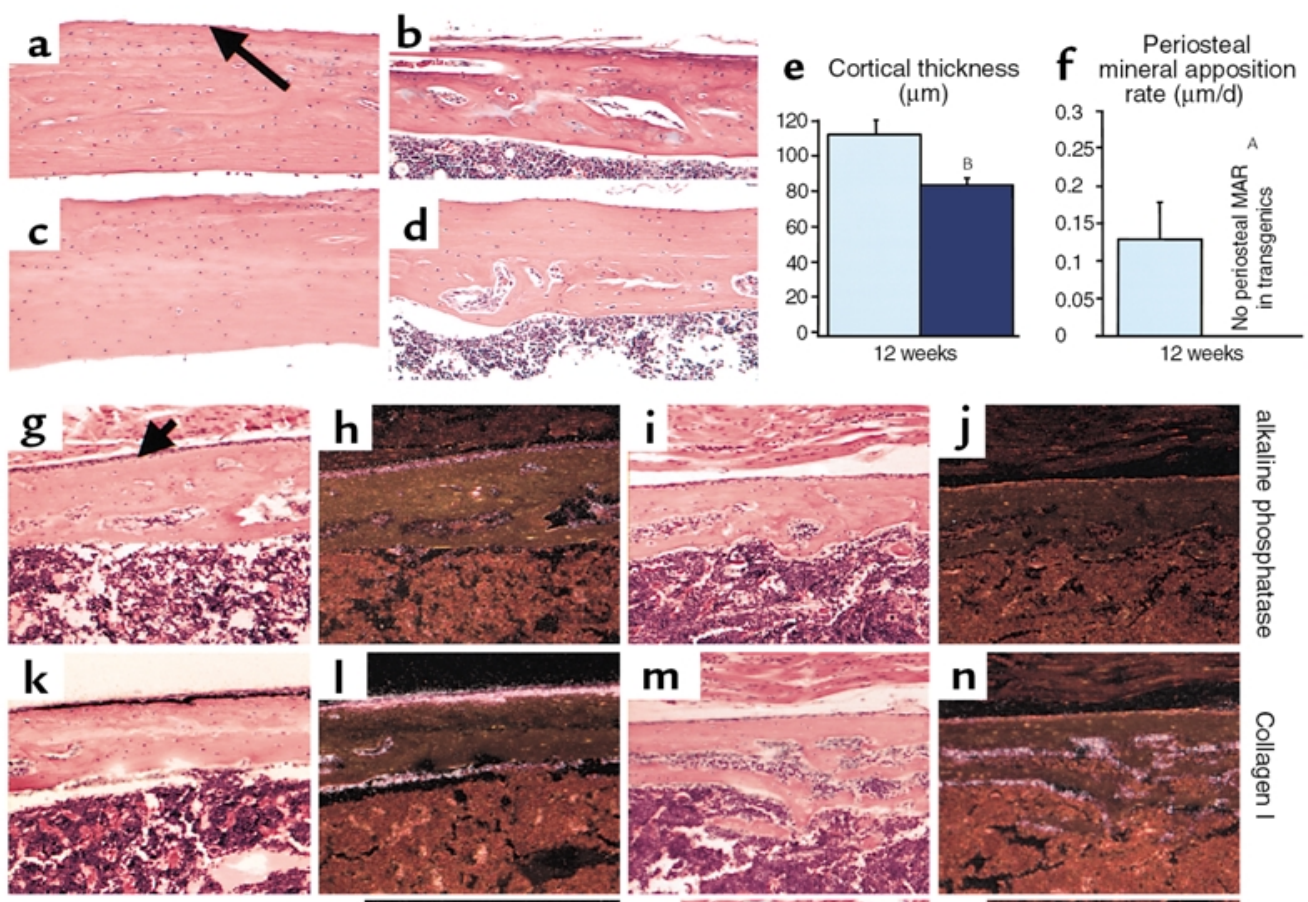

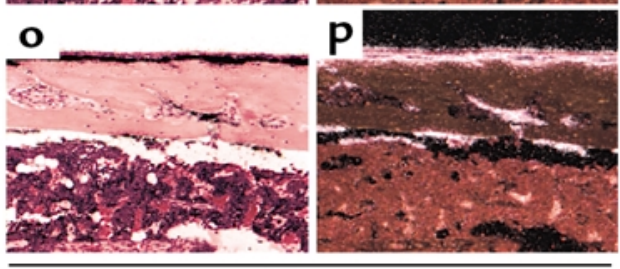

Wild-type

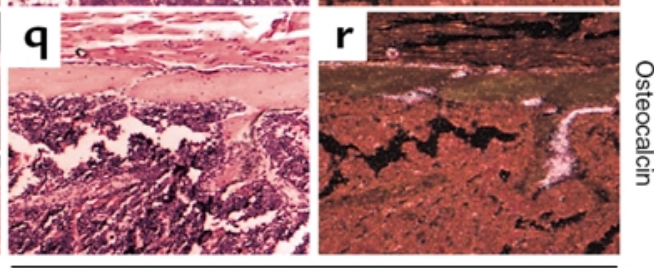

CL2 Transgenic

\section{Figure 5}

Analysis of cortical bone. (a-d) Histologic sections of cortical bone from tibial mid-diaphysis of 8-week-old wild-type (a) and CL1 transgenic (b) littermates and 12-week-old wild-type (c) and CL2 transgenic (d) littermates. (e and f) Histomorphometric analysis performed in 12-week-old wild-type (light blue) and CL2 (dark blue) littermates. ${ }^{A} P<0.05,{ }^{B} P<0.01$. Error bars represent the SEM. MAR, mineral apposition rate. (g-r) High-power $(\times 200)$ light microscopy images of the mid-diaphysis of in situ hybridization with the ${ }^{35}$ S-labeled alkaline phosphatase $(\mathbf{g}-\mathbf{j})$, collagen I (k-n), and osteocalcin (o-r) cRNAs in serial sections of decalcified proximal tibia of 2-week-old wild-type ( $\mathbf{g}, \mathbf{h}, \mathbf{k}, \mathbf{I}, \mathbf{o}, \mathbf{p})$ and CL2 (i, j, $\mathbf{m}$, $\mathbf{n}, \mathbf{q}, \mathbf{r})$ mice; sections were counterstained with hematoxylin and eosin. Bright-field $(\mathbf{g}, \mathbf{i}, \mathbf{k}, \mathbf{m}, \mathbf{o}, \mathbf{q})$ and dark-field (h, $\mathbf{j}, \mathbf{l}, \mathbf{n}, \mathbf{p}, \mathbf{r})$ views are shown. The arrow indicates the periosteal surface. 


\section{Figure 6}

Skull histology and histomorphometry. (a and $\mathbf{b})$ Sections of skulls from 12-week-old wild-type (a) and CL2 (b) littermates, stained with the method of Von Kossa. Arrows show the endosteal surfaces. (c-e) Histomorphometric analysis of the skull from wild-type (light blue) and CL2 (dark blue) littermates at 12 weeks of age. ${ }^{A} P<0.05$, ${ }^{\mathrm{B}} P<0.01$. Error bars represent the SEM.
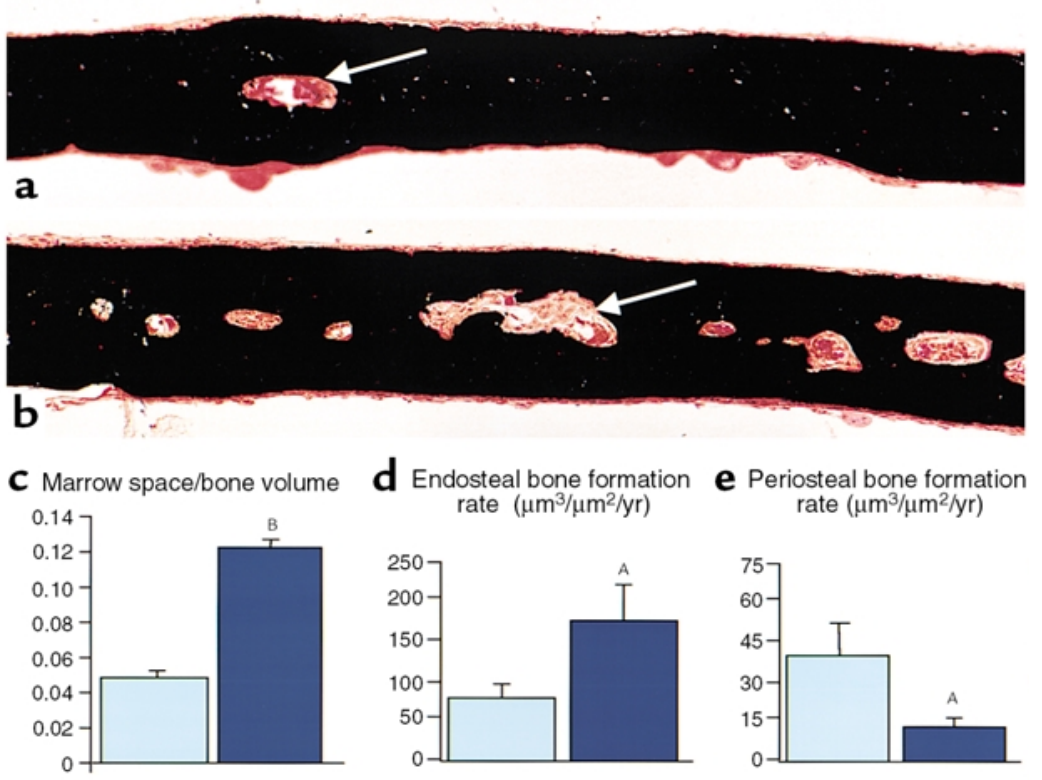

Increased bone remodeling on the endosteal surface and increased "porosity" was also observed in compact bone in the skull, which is formed by intramembranous ossification rather than the endochondral process by which long bones are formed. While calvarial thickness was unchanged in transgenic mice (wild-type thickness, micrometers: $86.0 \pm 4.5$; CL2: $87.4 \pm 5.5$ ), the transgenic calvaria were considerably more porous and contained more woven bone than wild-type calvaria in the same area (Figure 6, a and b). Quantification revealed an increase in the ratio of marrow space to bone volume in the calvarial bone in transgenic mice (Figure 6c). As observed in long bones, bone formation rate on the calvarial endosteal surfaces was significantly increased (Figure 6d), whereas the bone-formation rate on the calvarial periosteal surfaces was reduced (Figure 6e). The calvarial bone of CL2 mice also contained small areas of fibrosis in marrow spaces distant from the sagittal suture.

\section{Discussion}

To study in vivo the role of the PPR in cells of the osteoblast lineage, we generated mice that express the human mutant PPR, HKrk-H223R, under the control of the $2.3-\mathrm{kb}$ fragment of the mouse $\alpha 1$ (I) collagen promoter. This fragment has been shown previously to drive expression specifically in cells of the osteoblast lineage and in odontoblasts (14). The HKrk-H223R mutant was chosen rather than the other two mutant receptors described in JMC because of its higher levels of ligand-independent cAMP accumulation (12).

Specific expression of the transgene could be detected already at birth in transgenic mice. However, a change in bone structure was noted only starting from 1 week of postnatal age. Previous animal models have shown the important role of the PPR in fetal bone development (8). The lack of a clear phenotype at birth may be due to insufficient levels of prenatal expression of the transgene or to insufficient time after transgene activation to detect accumulation of abnormal cells and matrix.

Expression of transgene mRNA was detected at similar levels in trabecular bone and at both the periosteal and endosteal surfaces of cortical bone. This pattern of localization overlapped with that of the native PPR mRNA in bone (11), even if, as is different from the latter, it was more diffuse. These data are consistent with expression of the transgene being driven by a fragment of the collagen gene promoter. In addition, low but detectable expression of the transgene mRNA was present in the growth plate, and this might have contributed to the shortening of the limbs seen in the CL2 mice.

The actions of PTH in bone, while extensively studied $(6,7,20)$, are still controversial. PTH has been shown to either stimulate or inhibit the growth of osteoblastic cells and exert differentiating and dedifferentiating effects, depending on the model system studied (21-23). This study shows, we believe for the first time, that activation of the PPR in cells of the osteoblast lineage stimulates a vivid anabolic response in trabecular bone. This finding is consistent with the decreased trabecular bone seen prenatally in mice lacking the PPR (8). The increase in trabecular bone volume was related to an increase in both osteoblast number and function. In addition, an abundant population of stroma-like cells was noted among the trabeculae of the transgenic bones. In situ hybridization suggested that these cells are likely to represent cells committed to the osteoblast lineage, since they express markers of osteoblast differentiation, such as alkaline phosphatase, osteopontin $(24,25)$, and collagenase 3 (18). Interestingly, a population of cells with fibrotic morphology was noted in trabecular bone from mice given a high dose of $\mathrm{PTH}(1-34)$ continuously (7) and is a pathognomonic finding in 
patients with severe hyperparathyroidism (26). Our study supports the hypothesis that the "fibrosis" described in these cases may be due to an expansion of cells of the osteoblast lineage.

Consistent with activation of the adenylate cyclase pathway by HKrk-H223R, the stroma-like cells in our transgenic mice were morphologically similar to the pathologic findings typical of polyostotic fibrous dysplasia of bone (27). These bone lesions are associated with activating mutation of the GNAS gene, leading to constitutive activity of $\mathrm{G}_{\mathrm{s}} \alpha(28)$. As in our mice, the cells isolated from fibrous dysplasia lesions were also identified as a heterogeneous population of preosteoblasts (28). However, in contrast with what we observe in our transgenic model, accumulation of cAMP in fibrous dysplasia severely inhibited the differentiation of preosteoblasts into fully mature osteoblasts (28). The difference between these two models could depend on many variables, including onset of transgene expression and relative levels of intracellular cAMP, as well as potential activation of additional signaling pathways. Furthermore, a partial maturational arrest in a subset of the heterogeneous preosteoblast population present in our transgenic model cannot be excluded.

The increased proliferation rate detected in the trabecular osteoblastic cells of our transgenic mice suggests that it is the activation of the PPR that mediates the proliferative effect of PTH reported previously (29). Interestingly, an increase in proliferation rate is also noted in cells from fibrous dysplasia lesions (27). In CL2 transgenic mice, the proportion of osteoblasts and stroma-like cells undergoing apoptosis was decreased compared with wild-type littermates, suggesting that the PPR mediates the recently described (20) antiapoptotic effect of PTH on cells of the osteoblast lineage. Therefore, in our transgenic model the increased osteoblast number was, at least in part, the result of both augmented proliferation and decreased apoptosis in cells of the osteoblast lineage. The rate of commitment of mesenchymal stem cells also contributes to the pool of osteoblastic cells, and some evidence (29) has suggested that PTH may increase this rate. Further studies are needed to investigate whether the commitment rate of mesenchymal cells is modified in our transgenic model.

In CL2 mice, endosteal and trabecular bone formation rates were increased, suggesting that there was an increase in osteoblast function at these sites. An increased bone formation rate was also observed at the endosteal surface of the skull, which is formed differently from the long bones through a process of intramembranous ossification. These data show that the PPR is able to mediate an anabolic action in osteoblasts present in skeletal compartments of different developmental origin.

In sharp contrast to the increased bone-forming activity in both trabecular bone and the endosteal surface of the skull, an inhibition of both proliferation and function was noted in periosteal osteoblasts of the CL2 mice. Similarly, bone formation rate was decreased in periosteal osteoblasts from the skulls of transgenic mice. CL2 transgenic animals have therefore a dramatically different phenotype in the periosteal compartment when compared with the trabecular and endosteal compartments. Interestingly, similar levels of transgene mRNA expression were seen by in situ hybridization in these compartments. These findings could be helpful in explaining the differential effects of PTH in trabecular versus cortical bone in primary hyperparathyroidism (2). Changes in periosteal activity may play a role in the decreased cortical thickness in the CL2 transgenic animals, as well as in the increased cortical thickness seen in mice lacking the PPR (8). Additional experimental models are needed in order to investigate whether the differences among cells of the osteoblast lineage in these separate districts are due to intrinsic characteristics of the cells or to different bone microenvironments.

One of the most prominent actions of PTH is to increase, albeit indirectly, number and activity of osteoclasts $(2,7)$. By histologic and histomorphometric analysis, mature osteoclasts were increased dramatically in the trabecular and cortical compartments of our transgenic animals. Consistent with the increased number of osteoclasts, there was increased porosity of the cortical bone from transgenic mice. In addition, transgenic mice were normocalcemic, but had partially suppressed levels of circulating PTH, implying increased calcium release from bone. Therefore, expression of the constitutively active PPR solely in cells of the osteoblast lineage was sufficient to increase dramatically the number of mature osteoclasts in the transgenic mice. This fact points out that expression of this receptor in osteoblasts/stromal cells is critical for PTH-induced osteoclastogenesis, as has been suggested by in vitro evidence (30).

In summary, targeted expression of the constitutively active PPR led to increased osteoblast function in trabecular bone and at the endosteal surface of cortical bone, while the osteoblastic activity in the periosteum was inhibited. Mature osteoblasts, as well as a heterogeneous population of preosteoblasts, were increased in the trabecular compartment by a mechanism of increased proliferation and decreased apoptosis. Osteoblastic expression of the constitutively active PPR was sufficient to lead to a dramatic increase in osteoclast number. The net effect of these actions was a substantial increase in trabecular bone volume and a decrease in cortical thickness of the long bones.

Our data are dramatically different from the decreased net bone mass observed after continuous administration of PTH. It is plausible that dose of the peptide, as well as involvement of other signaling pathways and/or other receptors, play an important role in this catabolic effect of PTH. However, our findings, for the first time to our knowledge, identify the PPR as a crucial mediator of both bone-forming and boneresorbing actions of $\mathrm{PTH}$, as well as pointing out the complexity and heterogeneity of the osteoblast population and/or their regulatory microenvironment. 


\section{Acknowledgments}

This work was supported by the NIH grants DK-07028 (L.M. Calvi), AR-44855 (E. Schipani), and AR-45354 (R. Baron). We thank H. Jueppner and P.H. Carter for critical review of the manuscript and helpful discussion.

1. Karsenty, G. 1999. The genetic transformation of bone biology. Genes Dev. 13:3037-3051.

2. Parisien, M., et al. 1990. The histomorphometry of bone in primary hyperparathyroidism: preservation of cancellous bone structure. J. Clin. Endocrinol. Metab. 70:930-938.

3. Dobnig, H., and Turner, R.T. 1997. The effects of programmed administration of human parathyroid hormone fragment (1-34) on bone histomorphometry and serum chemistry in rats. Endocrinology. 138:4607-4612.

4. Bauer, W., Aub, J., and Albright, F. 1929. Studies in calcium phosphorus metabolism: study of bone trabeculae as ready available reserve supply of calcium. J. Exp. Med. 49:145-162.

5. Neer, R., et al. 2000. Recombinant human PTH [fhPTH (1-34)] reduces the risk of spine and non-spine fractures in postmenopausal osteoporosis. The Endocrine Society's 82nd Annual Meeting. Endocrine Society Press. Toronto, Canada. p. 42. (Abstr.)

6. Dempster, D.W., Cosman, F., Parisien, M., Shen, V., and Lindsay, R. 1993. Anabolic actions of parathyroid hormone on bone [erratum 1994 15:261]. Endocr. Rev. 14:690-709.

7. Uzawa, T., Hori, M., Ejiri, S., and Ozawa, H. 1995. Comparison of the effects of intermittent and continuous administration of human parathyroid hormone(1-34) on rat bone. Bone. 16:477-484.

8. Lanske, B., et al. 1999. Ablation of the PTHrP gene or the PTH/PTHrP receptor gene leads to distinct abnormalities in bone development. $J$. Clin. Invest. 104:399-407.

9. Jobert, A.S., et al. 1998. Absence of functional receptors for parathyroid hormone and parathyroid hormone-related peptide in Blomstrand chondrodysplasia. J. Clin. Invest. 102:34-40.

10. Karperien, M., et al. 1999. A frame-shift mutation in the type I parathyroid hormone (PTH)/PTH-related peptide receptor causing Blomstrand lethal osteochondrodysplasia. J. Clin. Endocrinol. Metab. 84:3713-3720.

11. Lee, K., Deeds, J.D., and Segre, G.V. 1995. Expression of parathyroid hormone-related peptide and its receptor messenger ribonucleic acids during fetal development of rats. Endocrinology. 136:453-463.

12. Schipani, E., Kruse, K., and Juppner, H. 1995. A constitutively active mutant PTH-PTHrP receptor in Jansen-type metaphyseal chondrodysplasia. Science. 268:98-100.

13. Parfitt, A.M., et al. 1996. Hypercalcemia due to constitutive activity of the parathyroid hormone (PTH)/PTH-related peptide receptor: comparison with primary hyperparathyroidism. J. Clin. Endocrinol. Metab. 81:3584-3588.

14. Rossert, J., Eberspaecher, H., and de Crombrugghe, B. 1995. Separate cisacting DNA elements of the mouse pro-alpha 1(I) collagen promoter direct expression of reporter genes to different type I collagen-produc- ing cells in transgenic mice. J. Cell Biol. 129:1421-1432.

15. Schipani, E., et al. 1997. Targeted expression of constitutively active receptors for parathyroid hormone and parathyroid hormone-related peptide delays endochondral bone formation and rescues mice that lack parathyroid hormone-related peptide. Proc. Natl. Acad. Sci. USA. 94:13689-13694

16. Marzia, M., et al. 2000. Decreased c-src expression enhances osteoblast differentiation and bone formation. J. Cell Biol. 151:311-320.

17. Parfitt, A.M., et al. 1987. Bone histomorphometry: standardization of nomenclature, symbols, and units. Report of the ASBMR Histomorphometry Nomenclature Committee. J. Bone Miner. Res. 2:595-610.

18. Zhao, W., Byrne, M.H., Boyce, B.F., and Krane, S.M. 1999. Bone resorption induced by parathyroid hormone is strikingly diminished in collagenase-resistant mutant mice. J. Clin. Invest. 103:517-524.

19. Stein, G.S., Stein, J.L., van Wijnen, A.J., Frenkel, B., and Montecino, M. 1996. Mechanisms regulating osteoblast proliferation and differentiation. In Principles of bone biology. J.P. Bilezikian and G.A. Rodan, editors. Academic Press. San Diego, California, USA. 69-86.

20. Jilka, R.L., et al. 1999. Increased bone formation by prevention of osteoblast apoptosis with parathyroid hormone. J. Clin. Invest. 104:439-446.

21. Bellows, C.G., Ishida, H., Aubin, J.E., and Heersche, J.N. 1990. Parathyroid hormone reversibly suppresses the differentiation of osteoprogenitor cells into functional osteoblasts. Endocrinology. 127:3111-3116.

22. Onyia, J.E., Bidwell, J., Herring, J., Hulman, J., and Hock, J.M. 1995. In vivo, human parathyroid hormone fragment (hPTH 1-34) transiently stimulates immediate early response gene expression, but not proliferation, in trabecular bone cells of young rats. Bone. 17:479-484.

23. Ishizuya, T., et al. 1997. Parathyroid hormone exerts disparate effects on osteoblast differentiation depending on exposure time in rat osteoblastic cells. J. Clin. Invest. 99:2961-2970.

24. Ducy, P., Zhang, R., Geoffroy, V., Ridall, A.L., and Karsenty, G. 1997. Osf2/Cbfa1: a transcriptional activator of osteoblast differentiation. Cell. 89:747-754.

25. Aubin, J.E., Liu, F., Malaval, L., and Gupta, A.K. 1995. Osteoblast and chondroblast differentiation. Bone. 17:77S-83S.

26. Pyrah, L.N., Hodgkinson, A., and Anderson, C.K. 1966. Primary hyperparathyroidism. Br. J. Surg. 53:245-316.

27. Marie, P.J., de Pollak, C., Chanson, P., and Lomri, A. 1997. Increased proliferation of osteoblastic cells expressing the activating Gs alpha mutation in monostotic and polyostotic fibrous dysplasia. Am. J. Pathol. 150:1059-1069.

28. Bianco, P., et al. 1998. Reproduction of human fibrous dysplasia of bone in immunocompromised mice by transplanted mosaics of normal and Gsalpha-mutated skeletal progenitor cells. J. Clin. Invest. 101:1737-1744.

29. Nishida, S., et al. 1994. Increased bone formation by intermittent parathyroid hormone administration is due to the stimulation of proliferation and differentiation of osteoprogenitor cells in bone marrow. Bone. 15:717-723.

30. Liu, B.Y., et al. 1998. Conditionally immortalized murine bone marrow stromal cells mediate parathyroid hormone-dependent osteoclastogenesis in vitro. Endocrinology. 139:1952-1964. 